\title{
Designing a Versatile Dedicated Computing Lab to Support Computer Network Courses: Insights from a Case Study
}

\author{
Gokhan Gercek and Naveed Saleem \\ University of Houston-Clear Lake, Houston, TX, USA
}

Gercek@uhcl.edu Saleem@uhcl.edu

\section{Executive Summary}

Providing adequate computing lab support for Management Information Systems (MIS) and Computer Science (CS) programs is a perennial challenge for most academic institutions in the US and abroad. Factors, such as lack of physical space, budgetary constraints, conflicting needs of different courses, and rapid obsolescence of computing technology, precipitate this challenge. While introductory computing courses can be adequately supported through general labs, advanced computing courses typically require dedicated labs. Normally, however, even a dedicated lab must simultaneously support multiple courses with diverse support requirements. Consequently, in designing dedicated computing labs, versatility and flexibility of the lab must be the prime consideration. Drawing on the insights gained from developing a dedicated lab, the authors present a model for developing a versatile, flexible lab to support computer network courses.

The proposed model entails a modular design approach which offers ease of management, configuration and upgrading of the lab by decoupling the lab functions into layers. The layered design divides the lab into four management layers: (1) wiring layer, (2) networking layer, (3) server systems layer, and (4) support procedures layer. Each layer offers a versatile and configurable design and serves as a foundation for the next higher layer.

Wiring layer minimizes the wire configuration efforts and enhances wiring flexibility by utilizing patch panels at the computer desks and the racks housing networking devices, and sufficient underground wiring between desks and racks. The underground wiring allows for network, telephone and serial cable connections as needed. Network layer builds LANs, subnets and internets with minimal configuration effort through a careful arrangement of network hubs, switches, routers, and firewalls. The network layer distinguishes between permanent networks needed in the lab, called pre-configured networks, and the networks that are assembled and disassembled by the students, called configurable networks. The network address allocation schemes for different types of network experiments are also discussed.

Material published as part of this journal, either on-line or in print, is copyrighted by the Informing Science Institute. Permission to make digital or paper copy of part or all of these works for personal or classroom use is granted without fee provided that the copies are not made or distributed for profit or commercial advantage AND that copies 1) bear this notice in full and 2) give the full citation on the first page. It is permissible to abstract these works so long as credit is given. To copy in all other cases or to republish or to post on a server or to redistribute to lists requires specific permission and payment of a fee. Contact Publisher@InformingScience.org to request redistribution permission.
Server systems layer covers two categories of servers: pre-configured servers and configurable servers. Pre-configured servers, such as DNS and file servers, are production servers that support network experiments. Configurable servers are hardware platforms that can be configured as needed according to experimental needs. The last layer, procedural layer, provides policies, guidelines and operational procedures that are needed 
for proper utilization of the lab space and the equipment. The paper also illustrates the application of the proposed model through the design and configuration of the computing lab for network courses underlying this case study.

Keywords: Designing Computing Labs, Multi-Purpose Dedicated Computing Lab, Versatile Dedicated Computing Lab

\section{Introduction}

During the past two decades, the computing function within organizations has evolved from a support to a strategic function that can provide a competitive edge to an organization. As a result, the need for technology-savvy personnel has increased exponentially, and colleges and universities have expanded their CS, MIS and related programs to meet this personnel demand. This growth, in turn, has resulted in the need for more faculty and support resources (Barker, 1999; Cherry, Phillabaum \& Valero, 2000; Madison, 2002). For many small US and international colleges and universities, introducing new computing programs and courses typically implies developing additional computing labs to support these programs. However, in developing these labs, the institutions normally encounter significant obstacles, such as budgetary constraints, lack of physical space to house the labs, conflicting computing support needs of different courses, and rapid obsolescence of technology (Borkowski, Elvove, Higgins \& Kueppers, 2004; Jones, Tunc \& Cherry, 2000; Lovgren, 2001; Madison, 2002; Walters, 1993; Wilson 2002). These obstacles become even more pronounced in the case of dedicated computing labs. A dedicated computer lab for a course is warranted when a general lab cannot provide adequate computing support for the course (Agarwal, Critcher, Foley, Santi \& Sigle, 2001; Dubose, 2000; Hill, Carver, Humphries \& Pooch, 2001). Conceptually, a dedicated lab is designed to support a specific course; normally, however, such labs support multiple courses (Belles, Gorka \& Miller, 2002; Belles \& Miller, 2002; Corbesro, 2003; Wooley, 2003). Consequently, it is imperative that the design and configuration of an under-development lab must be (1) versatile - to accommodate diverse and conflicting computing needs, (2) flexible - to efficiently support different courses, and (3) modular - to easily incorporate new technologies or allow lab reconfiguration (Giebel, 1999; Madison, 2002; Weeden, Scarborough \& Bills, 2003; Wilson, 2002).

The Management Information Systems (MIS) program at the University of Houston-Clear Lake (UHCL) has faced similar challenges since its inception in mid 1990s. Recently, the department developed a dedicated lab to support graduate and undergraduate courses such as computer networking, computer network security, host security, client-server system design, and system administration. During the lab design and development process, many choices were made to ensure versatility, flexibility and modularity of the lab to support multiple courses simultaneously. Drawing on their experiences in the design and development of this lab, the authors present a model of

a computing lab to specifically support computer networking and related courses. This model presents both physical as well as logical layout design considerations. It is believed that this model will prove a helpful reference for institutions that are undertaking development of such new labs.

\section{Physical Layout of the Lab}

Figure 1 visually depicts the physical layout of the lab, and this section describes this layout in detail. The lab encompasses an approximately 2000 square foot area and is furnished with twenty-four desks each supporting one workstation, but capable of accommodating two separate workstations. With two seats per desk, the lab is capable of seating forty-eight students. The desks are arranged in a lecture-hall setting.

At the rear side of the lab, racks are installed to house networking equipment and devices such as routers, firewalls, switches, hubs, a telephone switch (PBX) and patch panels. Another rack is 
utilized to house servers to be built by students. Ample space in the racks is provided for installing additional equipment as needed. The rack-based equipment is kept in the lab for students to perform hands-on experiments.

There are two categories of server computers utilized in the lab. The first category of servers, housed in the lab racks, is intended to be configured and administered entirely by students for courses such as system administration and application server design (see server systems layer section). The second category of servers supports lab operations and holds files and information relevant to the lab operations. Consequently, only select faculty and IT staff administer these servers. These servers are physically secured against unintentional or intentional mishandling by unauthorized users, including students. A secured pre-configured server room, adjacent to the lab, with an approximate area of 150 square feet is allocated to house lab operations servers. This is depicted in Figure 1. Connectivity and configuration for both categories of servers are detailed in later sections.

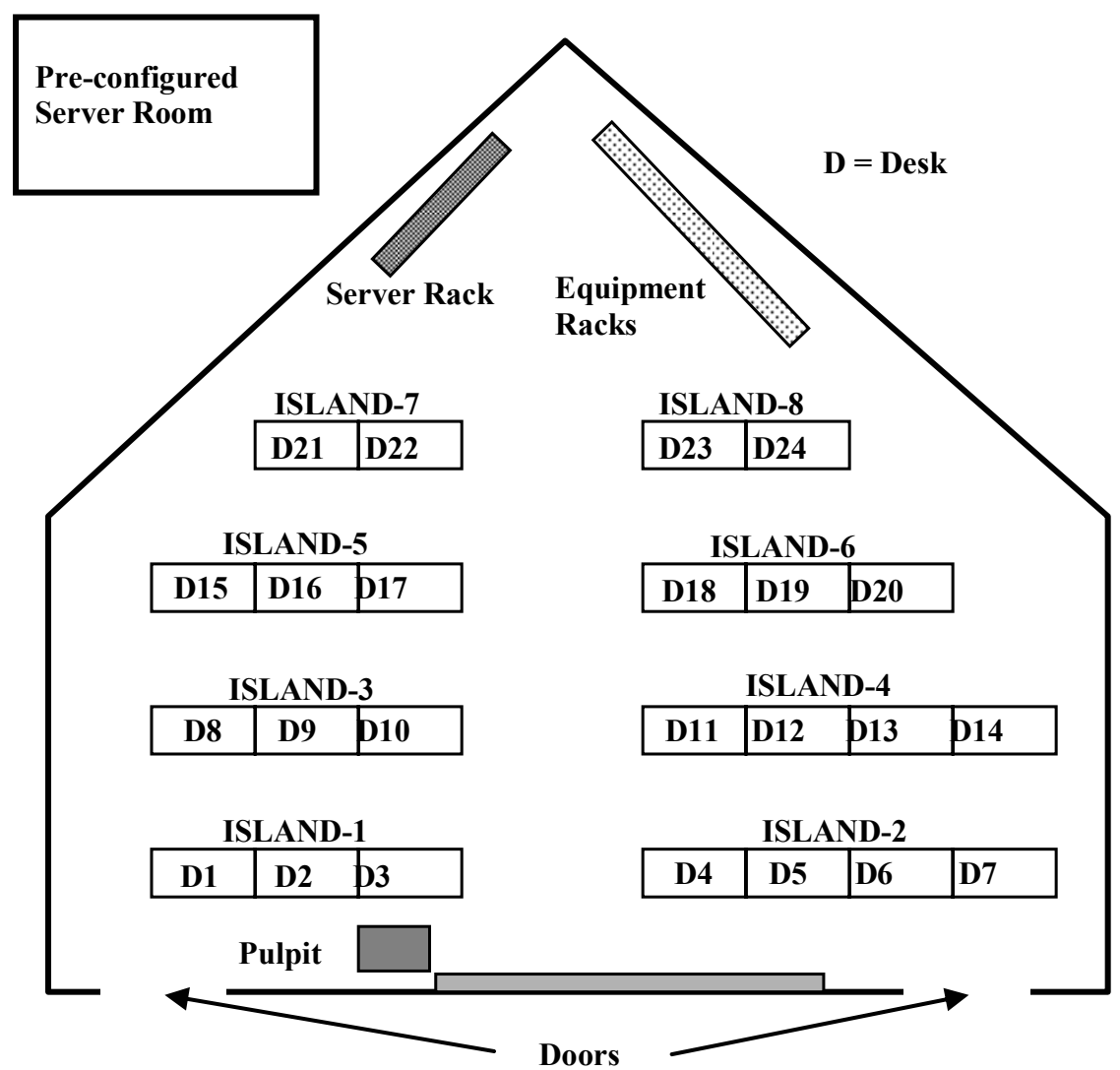

Figure 1: Physical Layout of the Lab

\section{Lab Design Methodology}

A computer network laboratory is expected to support as many as six different courses concurrently during long semesters. Consequently, the following objectives were sought in the lab design:

- The laboratory must provide as extensive support as possible to each course that it is intended to cover. 
- The laboratory must require minimal technician and support personnel involvement and intervention.

- The laboratory must require minimal, if any, switchover time for support from one course to another.

- Preferably, any switchover should be executable by students as part of their learning process.

- To the maximum extent, the laboratory should operate as an open lab supporting multiple courses at the same time.

- The laboratory must be as much "student-proof" as possible to eliminate possible downtimes that might arise from equipment mishandlings or procedural mistakes on the part of students.

- The laboratory should accommodate additional equipment and courses for growth.

- The laboratory must have growth accommodations for transition to newer technologies.

- The laboratory should require minimum or no rewiring and re-cabling for different types of courses and experiments

To achieve these objectives, a layered approach was employed for the design and development of the network laboratory. In the layered approach, each layer entails a related set of functional grouping. Every layer forms the underlying infrastructure for the next higher layer and, thereby, provides modularity, maintainability and upgradeability in the lab design. Specifically, this approach consists of the following layers: (1) wiring layer, (2) networking layer, (3) server systems layer, and (4) support procedures layer. These layers, shown in Figure 2, are described in the subsequent sections.

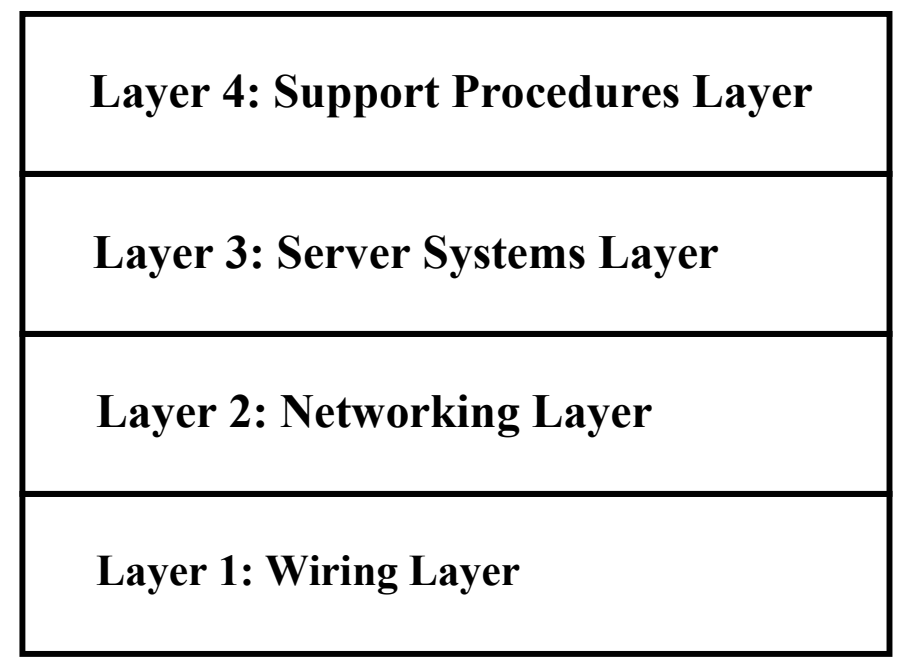

Figure 2: Network laboratory layered design approach

\section{Wiring Layer}

One of the key objectives sought in the network lab was to minimize loose cables between the desks and the networking equipment on the racks. The rationale behind this objective was twofold: First and foremost, loose wiring is a safety hazard and should be avoided as much as possible. Secondly, laying and removing wires between desks and racks would create significant amount of work for students during the experiments and would result in reduced student and lab 
efficiency. Consequently, a sufficient number of wires were pre-installed in hidden conduits under the floor between the desks and the racks. By use of patch panels and careful planning, the wiring management was reduced to a configuration at the patch panel level. Patch panel-based configuration provides significant flexibility, efficiency and effectiveness in configuring the laboratory for variety of experiments.

The desk-to-rack connectivity in the lab required three general categories of cables: network cabling, telephone cabling and serial-line cabling. Network cabling, implemented through four-wire twisted-pair cable connections from desks to the rack, provides Ethernet connectivity from desk computers to the routers, switches, hubs and servers on the equipment rack (see Figure 1). Importantly, this network cabling setup facilitates various types of network configurations such as desklevel networking, island-level networking and section-level networking, as shall be explained later.

Telephone cabling is required for analog telephony experiments. Telephone system design is a topic that is covered in data communications and networking courses. Consequently, a variety of experiments rely on a lab-based telephone system. A simulated telephone system environment is created using a Private Branch Exchange (PBX) telephone switch placed on the equipment racks. Cables for analog telephone lines are extended from desks to the equipment racks for experiments such as configuring PBX via administrative telephones, or simply using analog lines and PBX to simulate a Public Switched Telephone System (PSTN). PSTN simulation also allows modem connectivity between desk computers via the PBX. Such connectivity enables students to perform a variety of "dial-in" experiments such as Remote Access Server (RAS) design and dial-in routing.

Serial line cabling was installed to configure routers and switches using serial or console interfaces provided on those devices. In a typical experimental setting, a student sitting at his/her desk computer is able to access a rack-mounted router or switch using a serial cable and a terminal emulation program such as Microsoft Hyperterm. The serial cable allows connectivity between the serial port of a desk computer and the console port of a switch or router. A single serial cable is sufficient to perform serial cable-based experiments.

To allow for cross utilization of cables, four-pair (eight-wire) category-6 Ethernet cables were utilized for network, telephone, and serial line cabling. The number of cables needed for a variety of experiments was carefully analyzed and cable counts were formulated based on the desk arrangements of the laboratory.

As depicted in Figure 1, the desks in the laboratory are grouped in "islands" of four, three or two desks. Conduits were provided to carry cables from islands to the racks under the floor. Cables were placed in those conduits based on the needs formulated. The total number of category- 6 cables provided to each island was based on the formula $\mathrm{N}=2 \mathrm{D}+1$, where $\mathrm{N}$ is the total number of four-pair category- 6 cables and $\mathrm{D}$ is the number of desks in the island. To illustrate the use of the formula, an island with three desks requires a group of seven $(2 * 3+1)$ four-pair-wire cables installed in an under-the-floor conduit. Thus, two four-pair cables are dedicated to each desk. An additional four-pair cable is dedicated to the island for island-level networking. It should be remembered that each cable is capable of two Ethernet connections since Ethernet requires two pairs of wires. Two cables dedicated to each desk can be used in one of the following combinations:

- Two Ethernet and one serial connection to the desk

- One Ethernet, one telephone and one serial connection to the desk

- Two Ethernet and one telephone connection to the desk 
In summary, each desk is provided with two Ethernet connections, as one four-pair category- 6 cable allows for two Ethernet connections, and one serial or telephone connection via the second cable. As indicated earlier, a separate cable (island cable) is dedicated to allow Ethernet connectivity at the island level. When such connectivity is not needed, this cable can be used as additional Ethernet cabling for any of the desk computers. To achieve cabling versatility, standard RJ45 based patch panels were utilized at the racks and at the islands, as shown in Figure 3.

\section{To Switches and Routers}

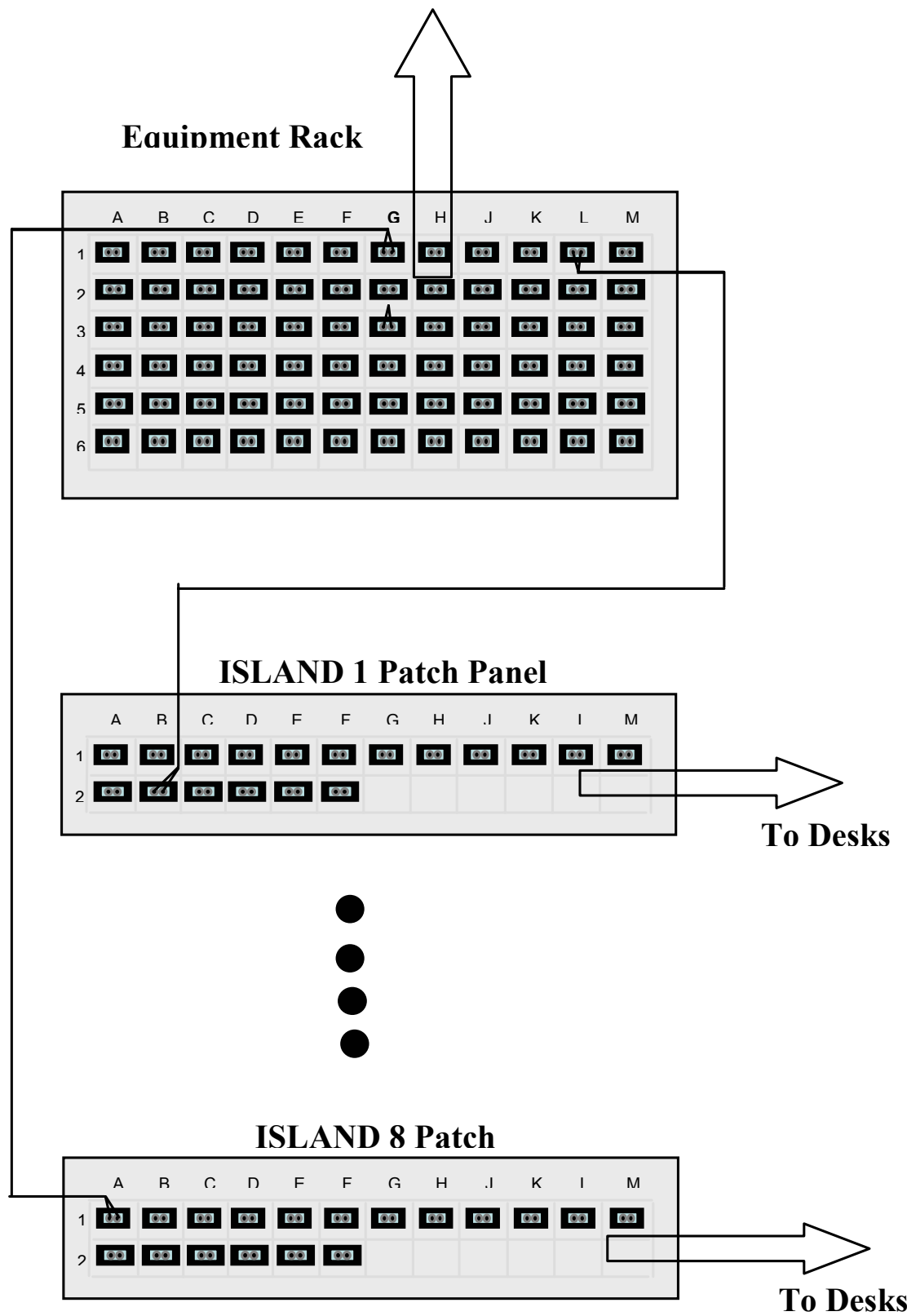

Figure 3: Patch-panel-based desk-island-rack-equipment connectivity 
Cables between the island patch panels and the rack patch panel were routed through the conduits under the floor. The cables between the island patch panel and the desk computers were routed in student accessible conduits under the desks.

To illustrate how a student can easily implement connectivity from a desk computer to a device, such as a router on the equipment rack, consider the following scenario: Assume that a student needs to connect his/her computer's Ethernet interface to a hub on the rack and also needs to connect the serial interface of the computer to the console interface of a router on the rack. First, the student would patch the two desk cables to designated ports on the island patch panel. Then he/she would locate the other ends of those two cables at the equipment rack patch panel and patch those ports to the hub and router of interest respectively. A student can complete such an operation in a matter of minutes.

\section{Networking Layer}

The networking layer consists of a variety of hardware and software-based products, such as Ethernet hubs, Ethernet switches, routers, CSU/DSUs, PBXs, firewalls and network management systems. The network layer aims at simulating secure or non-secure internets and intranets found in real world settings. Network layer connectivity is provided for two distinct categories of courses:

- Courses that involve network configuration experimentation where students are expected to configure and interconnect network layer devices such as Ethernet switches, routers and firewalls.

- Courses that involve experimentation such as designing and building server systems and client systems that require pre-configured network layer connectivity.

Consequently, the lab allows for two different types of networks: pre-configured networks and configurable networks.

A pre-configured network is comprised of a small internet built by using five routers. Each router supports two Ethernet interfaces and two Wide Area Network (WAN) interfaces as shown in Figure 4. Interconnectivity among the routers is achieved via WAN ports simulating a real Internet environment. Eight Ethernet interfaces provide Internet Protocol (IP) subnet level connectivity to eight islands, making each island a separate subnet simulating a corporate network environment. Thus, each island can be treated as a small corporate network with each desk computer representing a server or a client on the corporate network. Students can configure their computers as either servers or clients. The desk computers support removable hard drives allowing each student to load the intended operating system and application software for the purpose of the experiment. When the experiment period is over, students are required to turn off their computers, remove the hard disks from the computers and restore the island connectivity to its neutral setting by removing all the patches from the patch panels.

The fifth router (Router-5, Figure 4) has two subnets on its two Ethernet interfaces that allow placement of two groups of servers on the pre-configured network. The first group consists of pre-configured servers (see server systems layer section) that simulate a variety of publicly accessible servers found on the Internet. These servers are needed to complement various experiments by students. For example, one of the pre-configured servers can be used as a certificate server allowing students to build secure web servers utilizing https (secure hyper text transfer protocol) interactions among different islands. The second group of servers is made up of configurable servers (see server systems layer section) that are intended to be built and configured by students for various client-server experiments. 


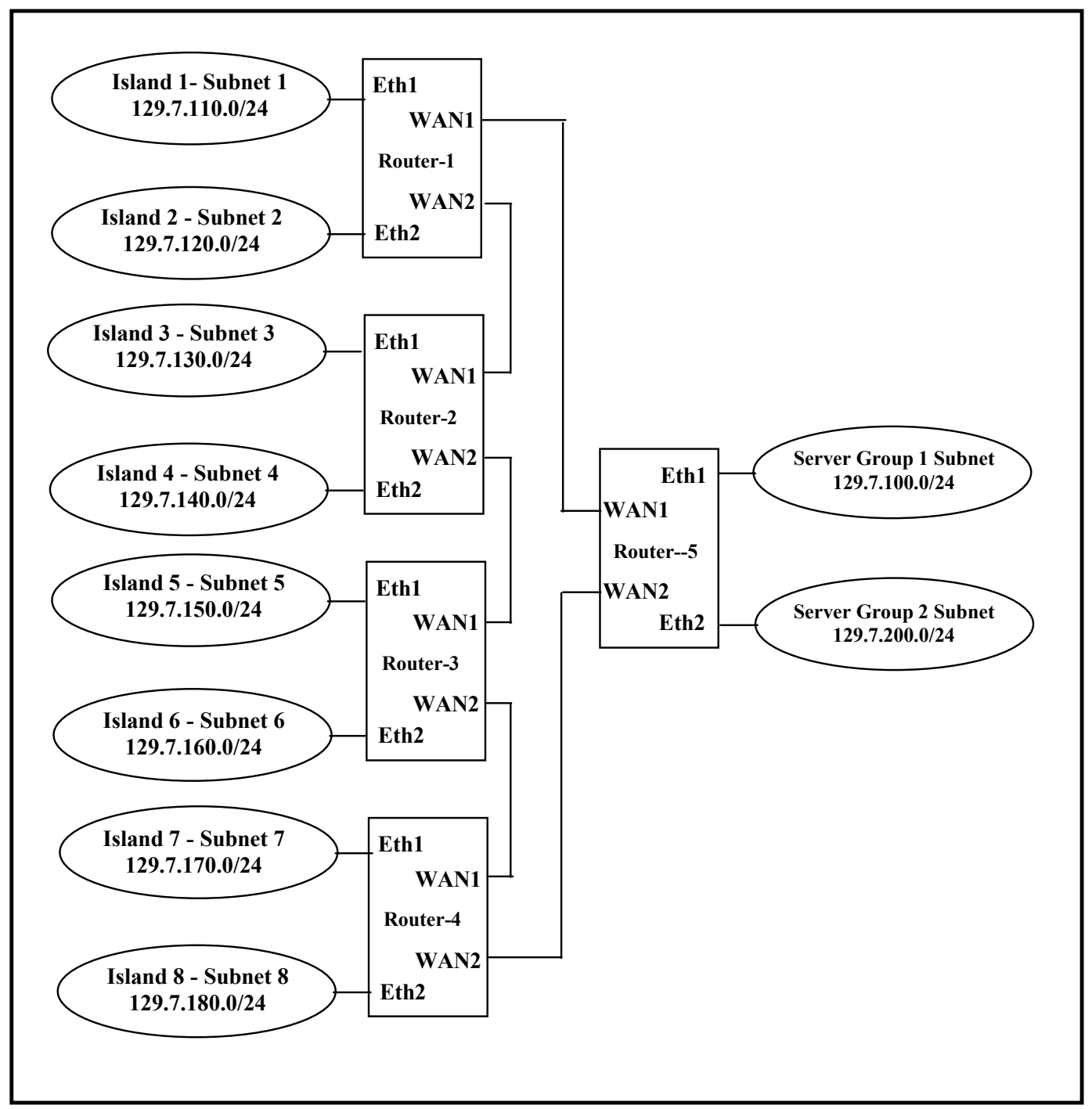

Figure 4: Pre-configured network setup in the lab

The connectivity of desktop computers to the pre-configured network is illustrated in Figure 5. The Ethernet wiring between each Ethernet interface on island routers and the corresponding island is provided via one of the two pairs of the "island cables" explained earlier. Figure 5 illustrates the connectivity from the desktop computers to the Ethernet interface of a router at the rack. To allow each desk computer to connect to the same interface of a router, an Ethernet hub is dedicated to each island for pre-configured network arrangement. Desk computers are connected to the hub via short Ethernet cables. The hub is connected to the island port on the patch panel that, in turn, is connected to the router. Figure 5 illustrates a typical island level pre-configured subnet.

In order to allow maximum flexibility with experiments, IP addresses for the configurable and pre-configured networks must be selected with careful planning. It is emphasized that for many experiments, two categories of networks are used as disjoint networks. In such cases, there is no need to provide interconnection between pre-configured and configurable networks. At other times, however, there may be need to interconnect the two types of networks. One such example is when students need to access the servers on the pre-configured network from configurable net- 


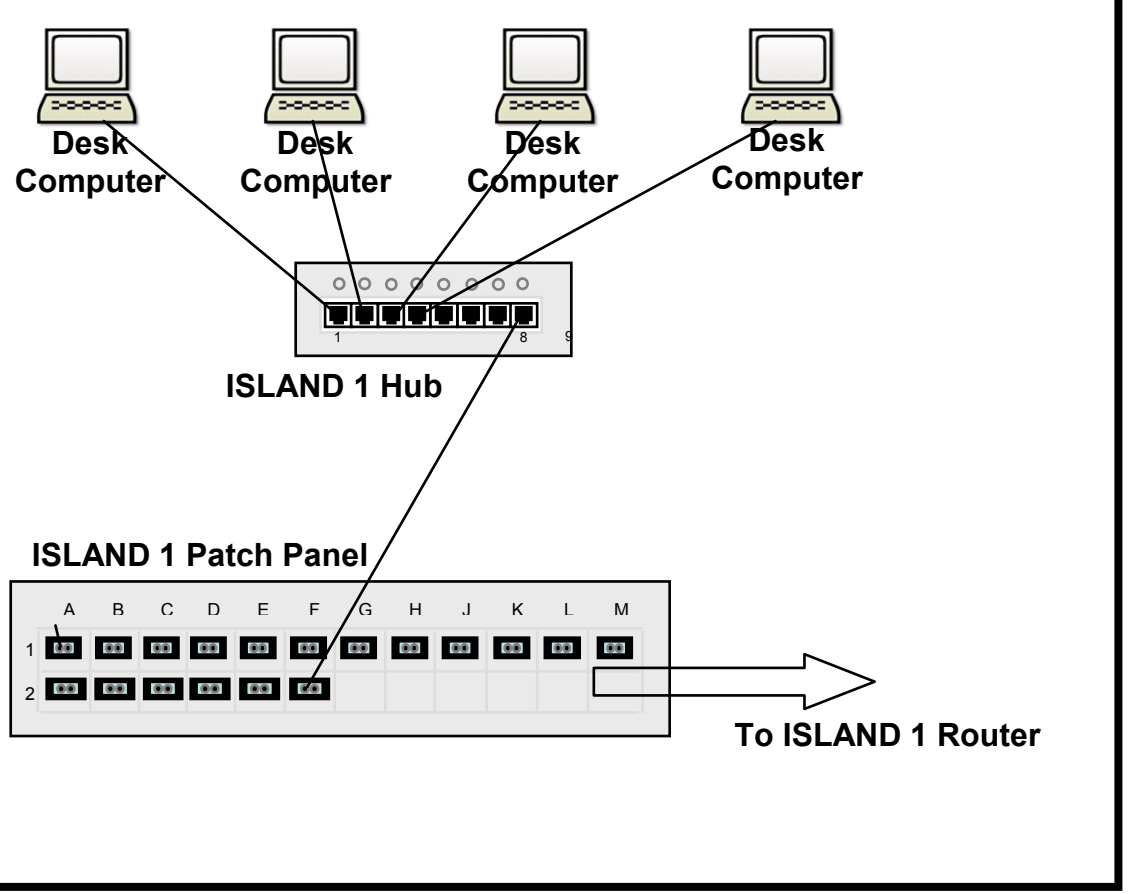

Figure 5: Pre-configured network connectivity at the island level

work hosts and devices. In order to avoid duplicate addresses, careful pre-allocation of IP addresses to both categories of networks is critical.

Table 1 and Table 2 show IP address allocations for pre-configured and configurable networks respectively. The IP address assignments shown in Table 1 are the same assignments as shown in Figure 4. For all IP assignments the subnet mask 255.255 .255 .0 or " $/ 24$ " is utilized.

Table 1: IP address allocation for the Pre-configured networks

$\begin{array}{ccc}\text { ISLAND } & \text { Subnet Address } & \text { Subnet Mask } \\ \text { Island 1 } & 129.7 .110 .0 & 255.255 .255 .0 \\ \text { Island 2 } & 129.7 .120 .0 & 255.255 .255 .0 \\ \text { Island 3 } & 129.7 .130 .0 & 255.255 .255 .0 \\ \text { Island 4 } & 129.7 .140 .0 & 255.255 .255 .0 \\ \text { Island 5 } & 129.7 .150 .0 & 255.255 .255 .0 \\ \text { Island 6 } & 129.7 .160 .0 & 255.255 .255 .0 \\ \text { Island 7 } & 129.7 .170 .0 & 255.255 .255 .0 \\ \text { Island 8 } & 129.7 .180 .0 & 255.255 .255 .0\end{array}$

Table 2 shows the configurable network IP address allocation for Island 1 only. A similar address allocation method is used for the remaining seven islands as well. As shown in the table, subnet addresses are assigned to islands in blocks of nine subnets with subnet masks 255.255.255.0. 
Table 2: Configurable network IP address allocation for Island 1

$\begin{array}{ccc}\begin{array}{c}\text { Island 1 Preconfigured } \\ \text { Address }\end{array} & \begin{array}{c}\text { Supported Con- } \\ \text { figurable } \\ \text { Addresses }\end{array} & \begin{array}{c}\text { Subnet Masks fo } \\ \text { Configurable } \\ \text { Addresses }\end{array} \\ 129.7 .110 .0 & 129.7 .111 .0 & 255.255 .255 .0 \\ 129.7 .112 .0 & 255.255 .255 .0 \\ 129.7 .113 .0 & 255.255 .255 .0 \\ 129.7 .114 .0 & 255.255 .255 .0 \\ 129.7 .115 .0 & 255.255 .255 .0 \\ 129.7 .116 .0 & 255.255 .255 .0 \\ 129.7 .117 .0 & 255.255 .255 .0 \\ 129.7 .118 .0 & 255.255 .255 .0 \\ 129.7 .119 .0 & 255.255 .255 .0\end{array}$

In spite of the fact that allocated IP addresses are "public" IP addresses, both pre-configured and configurable networks are isolated networks with no connectivity to Internet or campus networks. Therefore, employing a public addressing scheme doesn't interfere with any other networks.

As stated earlier, certain types of experiments require interconnecting pre-configured and configurable networks. This is the case, for example, when students need to experiment with building and configuring firewall-protected networks as part of network security course experiments. Incidentally, in firewall experiments additional subnet addresses are needed for firewall-protected networks. In such cases, address contiguity between configurable and pre-configured networks would be desirable to implement simpler static routes in the pre-configured routing tables.

Figure 6 provides an example of how pre-configured and configurable networks can be simultaneously created at the island level and interconnected. The figure exhibits (1) one pre-configured network with fixed address assignments, and (2) two firewalled subnets built and configured by students. The subnet addresses 129.7.111.0 - 129.7.119.0 can be freely employed by students for a variety of experiments including firewall experiments. It should be noted that the firewalls and the router shown in Figure 6 are mounted on the rack in the back of the laboratory. The connectivity to these devices is provided by means of wiring arrangements and the patch panels described in the wiring layer section above.

To build and experiment with large internets and intranets, another block of IP addresses is needed. Such Internets and intranets are built by students as part of various network course experiments. Therefore, these internets and intranets belong to the configurable network category. The IP address range 129.7.201.0 through 129.7.254.0 is reserved for such internet and intranet experiments. The laboratory is equipped with fifteen Cisco routers to support this type of experiment. These routers are permanently mounted on the equipment racks and are configured by students. 


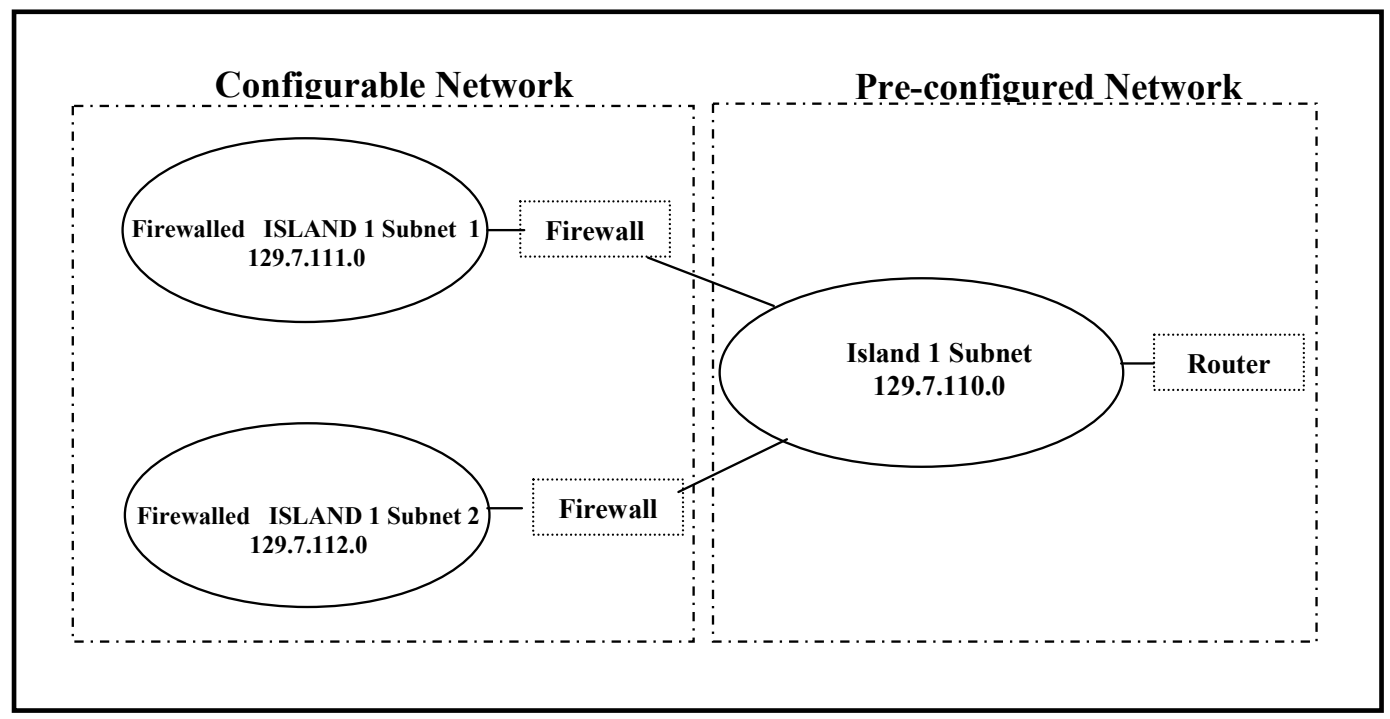

Figure 6: Interconnectivity between pre-configured network and configurable networks

When student-built internets need to be interconnected with pre-configured networks, the interconnectivity is provided via IP address 129.7.100.254. Thus, the pre-configured server network (see Figure 4) serves as a common network between configurable internets and pre-configured lab network.

From the description provided thus far, it should be clear that Layer 2 design allows network formations at three different levels: desk level, island level and sectional networking. Grouping one or more desks into a network creates desk networks. Grouping all desks in an island into a single network forms an island-level network. Grouping a number of islands into sections forms section-level networks. A commonly used section formation is achieved by grouping left-side and right-side islands of the lab into two different sections.

The network formations described above can also be created in a nested fashion if needed. First desks can be networked; then, networked desks can be integrated as subnets of island-level networks; island-level networks can in turn form subnets for section networks. Nested configuration flexibility allows experimentation for a variety of networking experiments.

\section{Server Systems Layer}

Layer 3 entails server systems. Server systems (1) support lab operations and (2) allow students to perform application server implementations of web servers, file servers, e-commerce servers and corporate server operations. Consequently, server systems are also grouped into two categories: pre-configured servers and configurable servers. Pre-configured servers (server group 1 in Figure 4) are lab support servers that are designed to simulate a variety of publicly accessible servers found on the Internet. These servers are configured and administered by faculty and staff. Consequently, students do not have any privileged access to these servers. The servers in this group include file servers for students to download files such as operating system images and configuration files, web servers that store experiment procedure instructions, NFS servers for UNIX experiments support, print servers to assist printing in the lab, and DNS servers to support networking experiments. Pre-configured servers are housed in the server room (see Figure 1) to provide physical security. 
The second group consists of configurable servers that are used for experiments by the students. These servers are built and administered by students and include file servers, e-commerce servers, domain controllers, domain servers, and NFS servers. The laboratory allows two types of computers to build configurable servers. Depending on the experiments, students may load their desk computers with necessary operating system and application software to build such servers or they may utilize dedicated configurable server computers located on the server rack at the back of the laboratory (see Figure 1). Since the desk computers are equipped with removable hard drives, each student can easily build and configure the desk computer as a server for the experiment and later remove the hard drive until the next lab session. Many server-level experiments involve students loading their desk computers and some rack servers with operating systems and server software to perform system and server administration experiments.

\section{Support Procedures layer}

Unlike the previous three, this layer is not a tangible layer. Since the network lab is a multipurpose open lab where different course experiments can be conducted simultaneously, policies, guidelines and operational procedures need to be clearly stated at the technical as well as at the procedural level (Delaney, Daigle \& Sparks, 2000). Students need to be well informed about how to resolve space and resource conflicts if they arise. Procedures need to be written in such a way that simultaneous experimentation is possible and the lab objectives specified earlier are realized. The lab procedures are kept in printed form in a folder in the lab and are also provided via the web server present in the laboratory for online viewing. At the beginning of semester, students are familiarized with the lab procedures prior to any experimentation. Since the lab procedures are dynamic documents that reflect lessons learned from experience and mistakes, they must be frequently updated and revised with feedback from students as well as from staff.

\section{Conclusion}

Providing adequate computing lab support is normally an ongoing challenge for academic institutions. Factors such as budgetary constraints, lack of physical space, conflicting needs of different courses, and rapid obsolescence of computing technology only exacerbate these challenges. In supporting specialized, advanced computing courses, these challenges become even more pronounced. In supporting such courses, the institutions primarily have two alternatives available to them: (1) design a dedicated lab for each course, and (2) design a versatile lab that can support multiple courses simultaneously. The first alternative is generally unfeasible for most institutions. The second alternative requires careful planning and a flexible lab design.

This paper presents a model of a versatile lab configuration that can support a number of computer networking and related courses within the same lab facility. This model presents a layered approach to lab design and configuration. Compartmentalizing various lab functions into different layers facilitates efficient operation, administration, and debugging and upgrade of hardware and software without impacting other lab support areas. Furthermore, separating the lab support functions into pre-configured and configurable groups assists experimentation and simplifies lab administration. Utilization of well-defined laboratory procedures allows efficient utilization, reconfiguration and maintenance of the laboratory. As an additional benefit, this design accommodates integrated experiments involving network courses as well as system courses such as network and system security experiments. Last but not least, the maintenance of up-to-date documentation of operational procedures removes any doubt and confusion on the part of students and administrative staff about various operating problems and their respective solutions. 


\section{References}

Agarwal, K. K., Critcher, A., Foley, D., Santi, R. \& Sigle, J. (2001). Setting up a classroom lab. The Journal of Computing in Small Colleges, 16(3), 281-286.

Barker, P. G. (1999). Using intranets to support teaching and learning. Innovations in Education and Training International, 36(1), 3-10.

Belles, R. \& Miller, J. R. (2002). Multi-purpose specialty labs: Providing specialized computer labs both cost and space effectively. The Journal of Computing Sciences in Colleges, 17(3), 46-52.

Belles, R., Gorka, S. \& and Miller, J. R. (2002). Flexible network topologies for your computing lab: VLAN and router technology at work in the classroom. The Journal of Computing in Small Colleges, $17(3), 53-59$.

Borkowski, E. Y., Elvove, J., Higgins, C., \& Kueppers, S. (2004). Voluntary centralization of user support in a de-centralized organization. Proceedings of the $32^{\text {nd }}$ Annual ACM SIGUCCS Conference on User Services, Baltimore, Maryland, 327-331.

Cherry, D., Phillabaum, P. \& Valero, P. (2000). Living on the bleeding edge: Creating and managing highly specialized labs. Proceedings of the $28^{\text {th }}$ Annual Conference on User Services, Richmond, Virginia, 40-49.

Corbesero, S. G. (2003). Teaching system and network administration in a small college environment. The Journal of Computing Sciences in Colleges, 19(2), 155-163.

Delaney, S., Daigle, R., \& Sparks, T. (2002). Discovering a new world of lab management. Proceedings of the $30^{\text {th }}$ Annual ACM SIGUCCS Conference on User Services, Providence, Rhode Island, 240-241.

Dubose, K. (2000). Manage all the computer labs on campus? What did I do to deserve this? Proceedings of the $28^{\text {th }}$ Annual ACM SIGUCCS Conference on User Services, Richmond, Virginia, 74-78.

Geibel, J. E. (1999). Administration of campus computing labs and faculty desktops. Proceedings of the $27^{\text {th }}$ Annual ACM SIGUCCS Conferences on User Services, Denver, Colorado, 71-78.

Hill, J. M. D., Carver, C. A., Jr., Humphries, J. W., \& Pooch, U. W. (2001). Using an isolated network laboratory to teach advanced networks and security. ACM SIGCSE Bulletin, 33(1), 36-40.

Jones, C., Tunc Y. \& Cherry, D. (2000). Approaches to computer lab management: Lockdown vs. freedom. Proceedings of the $28^{\text {th }}$ Annual ACM SIGUCCS Conference on User Services, Richmond, Virginia, 127-129.

Lovgren, T. D. (2001). Strategies for implementing unmanned labs. Proceedings of the $29^{\text {th }}$ Annual ACM SIGUCCS Conference on User Services, Portland, Oregon, 88-92.

Madison, D. E. (2002). A dynamic lab approach to reinforcing computing concepts. The Journal of Computing Sciences in Colleges, 17(3), 60-66.

Walters, J. E. (1993). Security in unattended computing labs: Safeguarding users as well as machines. Proceedings of the $21^{\text {st }}$ Annual ACM SIGUCCS Conference on User Services, San Diego, California, 267271.

Weeden, E. M., Scarborough, G. R. \& Bills, D. P. (2003). Lab management strategies for IT database curriculum. Proceedings of the ACM $4^{\text {th }}$ Conference on Information Technology Curriculum, Lafayette, Indiana, 16-18.

Wilson, J. H. (2002). Recipe to lab management or the cookie cutter approach to building labs. Proceedings of the $30^{\text {th }}$ Annual ACM SIGUCCS Conference on User Services, Providence, Rhode Islands, 298-300.

Wooley, B. A. (2003). Utilizing a computing lab to improve retention and recruiting of computer science and computer information science students. Journal of Computing Sciences in Colleges, 18(3), 228234. 


\section{Biographies}

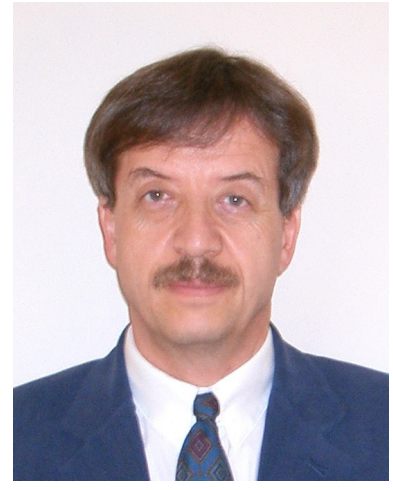

Gokhan Gercek is an Assistant Professor of Management Information Systems at School of Business, University of Houston-Clear Lake, Houston, Texas, USA. He received his Ph.D. in Electrical and Computer Engineering from Arizona State University in 1986, and joined the Electrical and Computer Engineering Department, Iowa State University, as a tenure-track faculty. In 1989, he joined Lockheed-Martin Corporation as a principal engineer in Houston, Texas, and worked at various NASA, USA, programs and projects through LockheedMartin. Furthermore, he also consulted in computer networking and computer security areas. In 2002, he joined University of HoustonClear Lake. Dr. Gercek has presented several research papers in professional conferences and seminars, and published in Academic Exchange Quarterly, Information Systems Security and Journal of Information Technology Education. His primary research interests include computer network design, network security, and network performance.

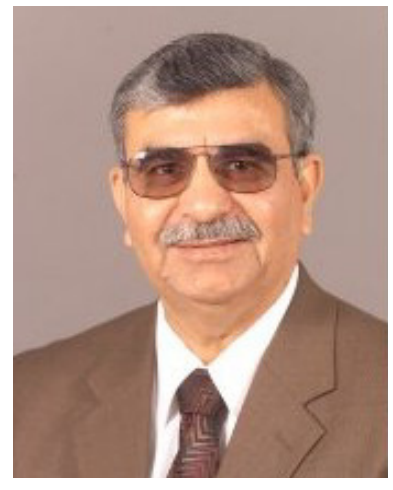

Naveed Saleem is Professor of Management Information Systems at the University of Houston-Clear Lake, Houston, Texas. He earned his Ph.D. in Management Science and Information Systems from the University of Texas at Austin in 1989. His research interests include system development strategies; database development, administration and security; web database applications; data warehousing; data mining; computer networks; and computer network security. His research papers have been published in Academic Exchange Quarterly, Computers in Nursing, Computer Personnel, EDP Audit and Security, Handbook of IS Management, Hospital Topics, Information Systems Security, International Journal of Commerce and Management, Journal of Business Disciplines, Journal of Information Technology Education, Journal of Management Information Systems, Journal of Nursing Administration, Management of End-User Computing, and Software Development Strategies. He is also Oracle Certified Professional as Application Developer, Database Administrator, Database Operator, and Oracle Internet Application Developer. 